\title{
Simultaneous acquisition of cerebral blood volume-, blood flow-, and blood oxygenation-weighted MRI signals at ultra-high magnetic field
}

Citation for published version (APA):

Krieger, S. N., Huber, L., Poser, B. A., Turner, R., \& Egan, G. F. (2015). Simultaneous acquisition of cerebral blood volume-, blood flow-, and blood oxygenation-weighted MRI signals at ultra-high magnetic field. Magnetic Resonance in Medicine, 74(2), 513-517. https://doi.org/10.1002/mrm.25431

Document status and date:

Published: 01/08/2015

DOI:

10.1002/mrm.25431

Document Version:

Publisher's PDF, also known as Version of record

Document license:

Taverne

Please check the document version of this publication:

- A submitted manuscript is the version of the article upon submission and before peer-review. There can be important differences between the submitted version and the official published version of record.

People interested in the research are advised to contact the author for the final version of the publication, or visit the DOI to the publisher's website.

- The final author version and the galley proof are versions of the publication after peer review.

- The final published version features the final layout of the paper including the volume, issue and page numbers.

Link to publication

\footnotetext{
General rights rights.

- You may freely distribute the URL identifying the publication in the public portal. please follow below link for the End User Agreement:

www.umlib.nl/taverne-license

Take down policy

If you believe that this document breaches copyright please contact us at:

repository@maastrichtuniversity.nl

providing details and we will investigate your claim.
}

Copyright and moral rights for the publications made accessible in the public portal are retained by the authors and/or other copyright owners and it is a condition of accessing publications that users recognise and abide by the legal requirements associated with these

- Users may download and print one copy of any publication from the public portal for the purpose of private study or research.

- You may not further distribute the material or use it for any profit-making activity or commercial gain

If the publication is distributed under the terms of Article 25fa of the Dutch Copyright Act, indicated by the "Taverne" license above, 


\title{
Simultaneous Acquisition of Cerebral Blood Volume-, Blood Flow-, and Blood Oxygenation-Weighted MRI Signals at Ultra-High Magnetic Field
}

\author{
Steffen N. Krieger, ${ }^{1,2 *}$ Laurentius Huber, ${ }^{1}$ Benedikt A. Poser, ${ }^{3}$ Robert Turner, ${ }^{1}$ \\ and Gary F. Egan ${ }^{2}$
}

Purpose: Yang et al. proposed an MRI technique for the simultaneous acquisition of cerebral blood volume (CBV), cerebral blood flow (CBF), and blood oxygenation level-dependent (BOLD)-weighted MRI signals (9). The purpose of this study was to develop modified version of the Yang sequence, which utilizes the advantages of 7 Tesla, leading to a robust and reliable MRI sequence.

Methods: The inversion recovery-based MR pulse sequence introduced here involves slice-saturation slab-inversion vascular space occupancy (SI-SS-VASO) MRI, double echo planar imaging readouts for arterial spin labeling, and VASO in order to correct for BOLD contamination, and a separate BOLD acquisition to minimize inversion effects on the BOLD signal. A standard visual stimulus block design was used to evaluate the spatial and temporal characteristics of CBV-, CBF-, and BOLD-weighted images.

Results: The high signal-to-noise ratio and spatial resolution of this method leads to robust activation maps. This technique enables the investigation of the differential spatial specificity and temporal characteristics of the different modalities.

Conclusion: The pulse sequence could be a powerful tool for studies of neurovascular coupling, hemodynamic response, or calibrated BOLD. Magn Reson Med 74:513-517, 2015. (C) 2014 Wiley Periodicals, Inc.

Key words: cerebral blood volume; cerebral blood flow; BOLD; ultra-high field; vascular space occupancy; VASO; functional MRI

\section{INTRODUCTION}

Today, several MRI techniques are used to map spatial and temporal patterns of functional activation. The blood oxygenation level-dependent (BOLD) contrast is the classic approach used in functional MRI (fMRI) at all field strengths (1). Cerebral neuronal activity is robustly detectable with high sensitivity, which makes fMRI available for a broad area of scientific research. The

\footnotetext{
${ }^{1}$ Max Plank Institute for Human Cognitive and Brain Sciences, Leipzig, Germany.

${ }^{2}$ Monash Biomedical Imaging, Monash University, Melbourne, Australia. ${ }^{3}$ Maastricht Brain Imaging Centre, Maastricht University, Maastricht, The Netherlands.

${ }^{*}$ Correspondence to: Steffen Krieger, Monash Biomedical Imaging, Building 220 Monash University, VIC 3800, Melbourne, Australia. E-mail: steffen. krieger@monash.edu

Received 5 March 2014; revised 5 August 2014; accepted 7 August 2014 DOI 10.1002/mrm.25431

Published online 5 September 2014 in Wiley Online Library (wileyonlinelibrary.com).
}

(c) 2014 Wiley Periodicals, Inc.

major drawback of BOLD contrast, however, is its poor spatial specificity. The BOLD signal is directly coupled to the concentration in deoxyhemoglobin of blood. Therefore, downstream draining veins can show large BOLD signal changes during neuronal activation, even when relatively far from the area of activation (2). Additionally, the fact that BOLD signal emerges from a combination of changes in cerebral blood flow (CBF), cerebral blood volume (CBV), and cerebral oxygen metabolism $\left(\mathrm{CMRO}_{2}\right)$ makes this contrast difficult to interpret. Furthermore, BOLD signal sensitivity to variations in baseline physiology limits its reproducibility and hampers fully quantitative analysis (3).

Alternative fMRI techniques use single physiological quantities as a marker of neuronal activity rather than a combination of many. Arterial spin labeling (ASL) is a noninvasive technique to measure $\mathrm{CBF}$, and various approaches have been introduced to provide optimal MR signal quality (4-6). ASL uses magnetically labeled blood water as an endogenous tracer; and in contrast with BOLD, it has a strong arteriolar and capillary signalweighting, which gives this technique an improved spatial specificity (7). The major problem, however, is its low signal-to-noise ratio (SNR). ASL relies on the MR signal coming from the blood, which typically contributes less than $5 \%$ to the total signal arising from each voxel (7). Vascular space occupancy (VASO) has been used to measure functionally induced changes in CBV (8). VASO also relies on the inversion of blood water spins, but in contrast with ASL, the MR signals are acquired at the blood nulling time point.

BOLD, ASL, and VASO show different signal characteristics with regard to spatial and temporal sensitivity and specificity. Simultaneous detection of multiple MRI signals could thus be useful to combine the advantages of each technique. Yang et al. recently introduced an MRI technique to simultaneously map BOLD-, CBF-, and CBV-weighted MRI signals at 3 Tesla (9). This technique has already found a series of applications (10-12), and several technical developments have followed the original proposal (13-16). Based on Yang et al., we have developed a new method to simultaneously measure CBV-, CBF-, and BOLD-weighted MRI signals optimized for ultra-high static magnetic field strength. The purpose of this study was to develop an MR sequence that overcomes several potential drawbacks of Yang et al. while utilizing the advantages of ultra-high magnetic fields.

The key modifications compared to the original technique developed by Yang et al. include the application of slice-saturation slab-inversion VASO (SS-SI-VASO), 


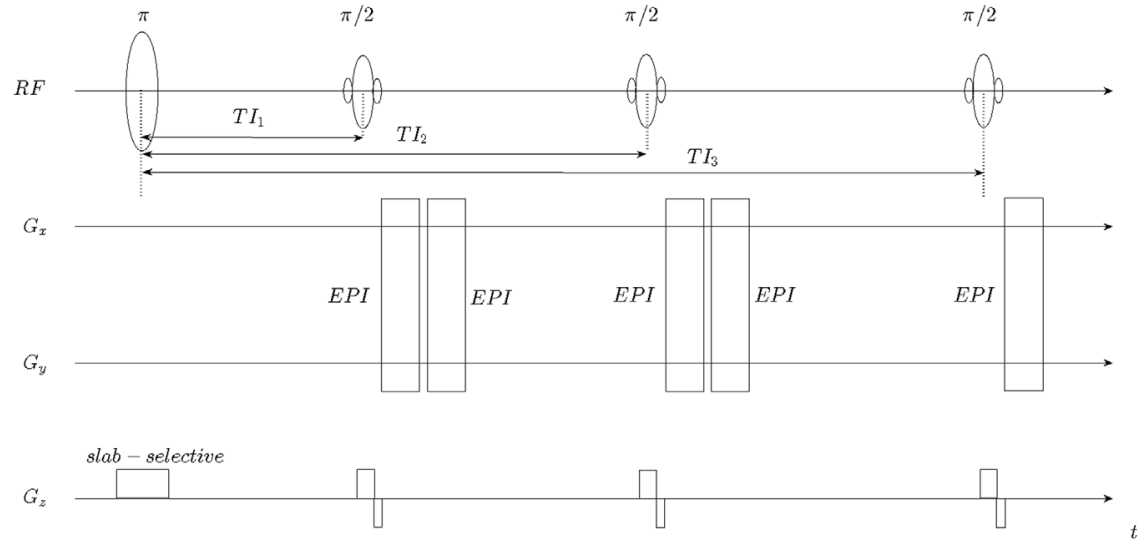

FIG. 1. Sequence diagram of simultaneous slice-saturation slab-inversion vascular space occupancy (after $\mathrm{TI}_{1}$ ), arterial spin labeling (after $\mathrm{Tl}_{2}$ ), and blood oxygenation level-dependent (after $\mathrm{Tl}_{3}$ ) signal acquisition at 7 Tesla. EPI, echo planar imaging.

providing increased SNR in the CBV-weighted images (17). Furthermore, multiple echo planar imaging (EPI) readouts are used to correct for BOLD signal contamination of the VASO and ASL images. High inversion efficiencies and sharp inversion profiles were achieved via an adiabatic inversion radio frequency (RF) pulse specifically designed for 7 Tesla (18). Additionally, a separate BOLD acquisition at the end of the sequence minimizes inversion effects on the BOLD signal. This technique can be used to study neurovascular coupling, quantification of oxygen metabolism, and to investigate biophysical models of the BOLD signal.

\section{METHODS}

\section{SS-SI VASO Signal Acquisition}

In order to obtain robust and uniform slab-selective inversion despite $B_{1}$ inhomogeneities and substantial specific absorption rate (SAR) constraints, a slab-inversion time resampled frequency offset corrected inversion (Tr-FOCI) pulse (18) was used to magnetically label blood water spins (pulse duration $=5.5 \mathrm{~ms}$, pulse amplitude $=400 \mathrm{~V}$, slab thickness $=142.8 \mathrm{~mm}$ ), as suggested by Huber et al. (17). At inversion time $(\mathrm{TI})_{1}=1330 \mathrm{~ms}$, a single slice positioned across the visual cortex was excited, followed by two EPI readouts providing two VASO images at two different echo times $\left(\mathrm{TE}_{\mathrm{VASO}, 1}=14 \mathrm{~ms}, \quad \mathrm{TE}_{\mathrm{VASO}, 2}=37 \mathrm{~ms}\right)$. Imaging parameters for all acquisitions were: field of view $=192 \times$ $192 \mathrm{~mm}$, nominal isotropic resolution $=1.5 \mathrm{~mm}$, generalized autocalibrating partially parallel acquisitions (GRAPPA) factor $=3$, partial Fourier factor $=6 / 8$, repetition time $(\mathrm{TR})=3$ seconds.

\section{ASL and BOLD Signal Acquisition}

The second excitation was performed at $\mathrm{TI}_{2}=1650 \mathrm{~ms}$, followed by two consecutive EPI readouts $\left(\mathrm{TE}_{\mathrm{ASL}, 1}=14\right.$ $\mathrm{ms}, \mathrm{TE}_{\mathrm{ASL}, 2}=37 \mathrm{~ms}$ ) giving $\mathrm{CBF}$-weighted MRI images. No additional inversion pulse was applied between VASO and ASL readout. However, the delayed acquisition of the CBF-weighted image provided enough time to have nonexcited blood flowing into the imaging slice, and thus avoid saturation effects due to earlier excitation RF pulses.

BOLD signals were acquired at $\mathrm{TI}_{3}=2250 \mathrm{~ms}$ in order to minimize the influence of earlier inversion pulses on the BOLD contrast. The echo time used for the BOLD image was $\mathrm{TE}_{\mathrm{BOLD}}=22 \mathrm{~ms}$, which provides optimal contrast with minimal susceptibility-induced void artifacts. The sequence diagram can be found in Figure 1.

\section{EXPERIMENTAL DESIGN}

The data were acquired in five healthy participants on a Siemens Magnetom 7 Tesla whole body MRI scanner (Siemens Healthcare, Erlangen, Germany) equipped with a 24-channel head coil (NOVA Medical Inc, Wilmington, MA). All participants gave written informed consent for participation in this study. Ethics approval was obtained from the local review board (Ethics Commission, Leipzig University). Visual stimulation with an 8-Hz black-andwhite radial checkerboard was presented on a screen inside the magnet with a standard block design of 30 seconds of rest, followed by 30 activation periods. The complete procedure took 8 minutes.

\section{Data Analysis}

VASO and ASL images were extrapolated to $\mathrm{TE}=0 \mathrm{~ms}$ using a single compartment model (19). Extrapolated VASO and ASL images and the BOLD images were then corrected for in-plane motion using the fMRI expert analysis tool v. 5.98 (Functional MRI of the Brain Software Library, Oxford University, UK) using no smoothing kernel. SNR and temporal SNR (tSNR) was calculated in gray matter for all subjects based on the extrapolated SS-SI-VASO and ASL images at $\mathrm{TE}=0 \mathrm{~ms}$. Clusterthresholded activation maps $(P<0.05$, multiple comparison corrected) for VASO, ASL, and BOLD signals were calculated using the same software. Time courses of significantly activated voxels were averaged in time using in-house analysis tools based on MATLAB (v. 8.0.0.783 [R2012b]; MathWorks, Natick, MA).

\section{RESULTS}

The average SNR in gray matter of the SS-SI-VASO and ASL images acquired via the simultaneous acquisition technique was $9.3 \pm 1.2$ and $14.3 \pm 3.0$, respectively. The corresponding tSNR of SS-SI-VASO and ASL in gray matter was $12.0 \pm 1.4$ and $18.5 \pm 2.1$, respectively. Statistical activation maps of CBV, CBF, and BOLD from all subjects are shown in Figure 2. The relatively high 


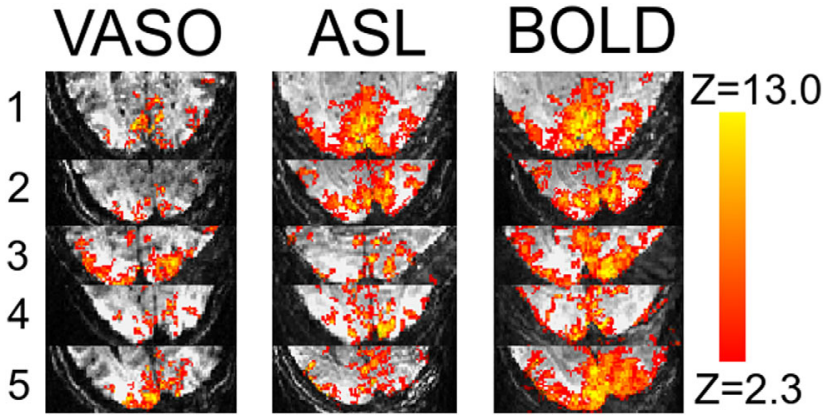

FIG. 2. Statistical activation maps of cerebral blood volume-, cerebral blood flow-, and BOLD-weighted MRI images of all subjects.ASL, arterial spin labeling; BOLD, blood oxygenation level-dependent; VASO, vascular space occupancy.

spatial resolution used in this study enables the investigation of the differential spatial specificity of the different modalities. Whereas BOLD contrast shows significant activation at the cortical surface, VASO and ASL signal changes appear to peak only within gray matter, as expected. The CBV and CBF activation maps were based on the VASO and ASL MRI images, when extrapolated to $\mathrm{TE}=0 \mathrm{~ms}$. Single-subject time courses of VASO, ASL, and BOLD signals averaged across all significantly activated voxels can be found in Figures $3 \mathrm{~A}, \mathrm{~B}$, and $\mathrm{C}$, respectively. Group averages of each quantity are plotted in the same graphs. The time courses have been normalized to the baseline of each quantity, respectively.

\section{DISCUSSION}

Based on the technique developed by Yang et al. (9), we have introduced an imaging technique to simultaneously acquire CBV-, CBF-, and BOLD-weighted MRI signals at ultra-high static magnetic field strength. The spatial and temporal SNR characteristics achieved in this study are comparable to those obtained in studies using the acquisition of a single quantity only at comparable spatial resolutions and field strengths $(17,20,21)$. As expected, BOLD-weighted signals showed larger areas of significant functional activation than did CBF- and CBV-weighted signals. BOLD signal changes were observed to occur not only at the site of neuronal activity but also in downstream venules and veins, consistent with the reliance of the BOLD signal on changes in oxy-deoxyhemoglobin ratio, which occur mainly on the venous vessel side $(2,22)$. In contrast, VASO and ASL signal changes originate mainly from arteries and capillaries because the highest fractional changes in cerebral blood volume and flow occur in these compartments. Presumably due to differences in the compartmental weighting of the VASO and the ASL signal, spatial differences in CBV and CBF activation maps are observable in Figure 2. Reduced partial volume effects because of the relatively high spatial resolution used in this study might highlight this effect. Furthermore, differences of blood $\mathrm{T}_{1}$ across subjects might also lead to variations in the extent and amplitude of regional brain activation in ASL and VASO statistical activation maps.

VASO and ASL suffer from an intrinsically lower sensitivity but provide increased spatial specificity as com- pared to BOLD contrast $(23,24)$. When sequentially acquired MR signals are compared and interpreted, motion and image artifacts are major concerns. Furthermore, attention and habituation effects have to be taken into account when comparing MR signals that are not acquired simultaneously because these may affect the neuronal and hemodynamic response to functional stimuli. The pulse sequence introduced in this article thus enables the study of brain function with combined high sensitivity and high specificity, which may provide insight into the biophysics of the neurovascular response-a major practical advantage compared with sequential signal acquisition.

Despite the advantage of providing increased MR signals compared to lower static magnetic field strengths, 7 Tesla MRI entails several challenges when an inversion recovery sequence is used. For example, a reduced difference in longitudinal relaxation times $\mathrm{T}_{1}$ of blood and tissue leads to small contrast-to-noise (CNR) of classic VASO (20), and positive BOLD signal changes

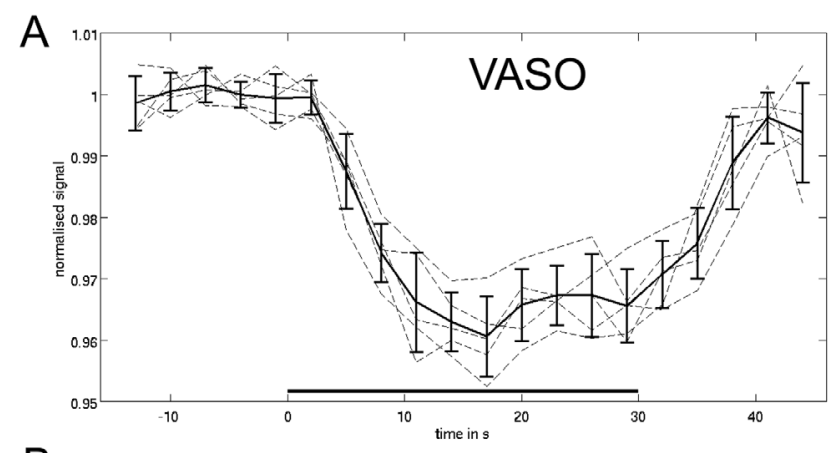

$B$

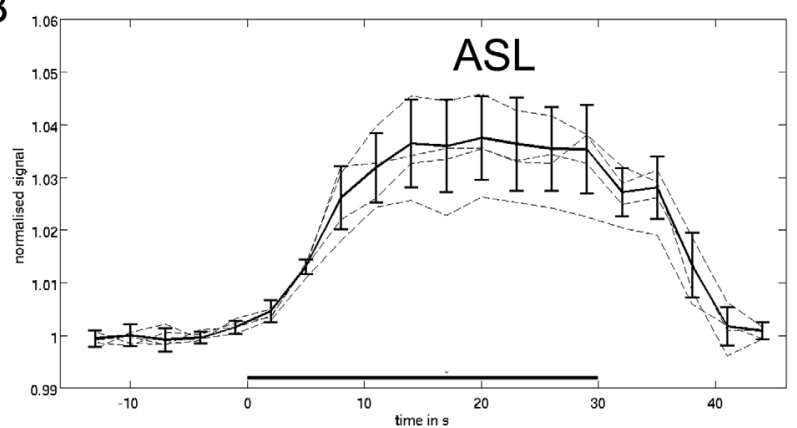

C

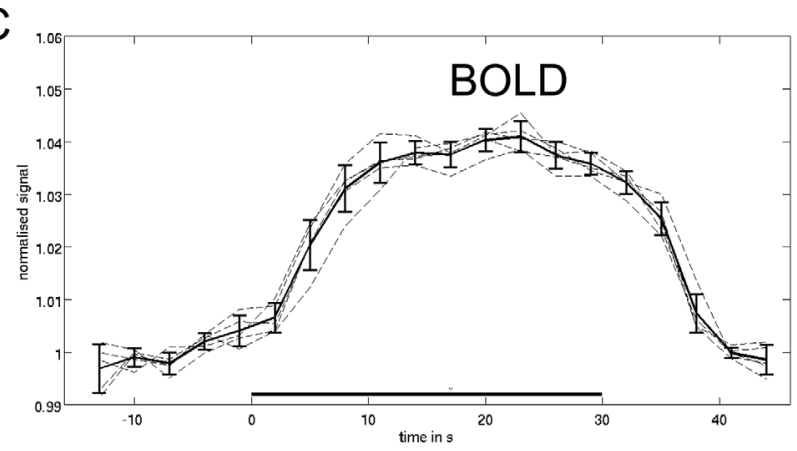

FIG. 3. Time courses of VASO (A), ASL (B), and BOLD (C) from all five subjects were normalized to baseline and plotted in dashed lines. The averaged time courses across subjects of VASO, ASL, and $B O L D$ are plotted as bold solid lines in $A, B$, and $C$, respectively. ASL, arterial spin labeling; BOLD, blood oxygenation level-dependent; VASO, vascular space occupancy. 
increasingly counteract the negative VASO signal change (19). Furthermore, RF field inhomogeneities and SAR limits hamper efficient spin inversion. Use of a tr-FOCI pulse with SS-SI VASO was found to overcome these obstacles (18) and increases SNR and CNR compared to classic VASO approaches. Slab-selective inversion enables labeled blood to be refreshed between each inversion pulse, which leads to a steady-state tissue signal, but a blood signal that has experienced only one inversion. The increased blood $\mathrm{T}_{1}$ at higher field strengths provides higher SNR due to the slower decay of the labeled blood spins. A potential problem of the current implementation of this technique is the inflow of fresh blood into the imaging slice before the VASO and the ASL signal have been acquired. Inflow of noninverted spins could introduce a CBF-weighting in the SS-SIVASO contrast. In future studies, a partial inversion technique could be applied to reduce the blood nulling time and therefore reduce the vulnerability to inflow effects (17). Furthermore, multislice acquisition could make this technique more robust against head motion because standard motion correction algorithms could be applied properly. In this study, only experienced subjects that are known to show little movement during the scanning sessions have been selected in order to avoid deviations resulting from head motion. A multislice extension of this technique would, however, be limited by the total acquisition time, especially with regard to the VASO blood nulling condition. In addition, energy deposition restrictions and effects on the ASL signal due to prior excitations would have to be assessed. Another potential drawback of the simultaneous acquisition of three physiological quantities is that advanced ASL techniques such as the application of saturation pulses to actively control the temporal width of the labeled bolus (25) and background suppression $(26,27)$ cannot be applied.

The technique introduced in this article involves three excitation pulses applied on one slice within one TR. Previous RF pulses might affect the ASL and the BOLD signal acquired at a later time point. Therefore, it is crucial to maximize the time between the acquisition of the three contrasts to allow the blood inside the excited slice to refresh before the next acquisition takes place and to avoid detrimental effects on the next acquisition. Longer TI reduces the ASL signal, however, due to $\mathrm{T}_{1}$ decay. We found optimal results for $\mathrm{TI}_{2}=1650 \mathrm{~ms}$ and $\mathrm{TI}_{3}=2250 \mathrm{~ms}$ while taking into account a fixed VASO inversion time. In order to minimize $\mathrm{T}_{1}$ - weighting associated with flow, the BOLD signal acquisition is performed at the end of the pulse sequence using a single RF excitation followed by a single EPI readout. This feature is especially necessary at high magnetic field strengths due to the increased longitudinal relaxation times of blood compared to lower field strengths.

The comparison and interpretation of simultaneously acquired signal time courses of CBV, CBF, and BOLD may become a powerful strategy for future studies of brain function. The spatiotemporal characteristics of VASO, ASL, and BOLD MRI signals have been found to rely on various parameters such as type of functional task, brain area, task duration, age, sleep, and awareness (28-32).
Simultaneous acquisition of multiple physiological parameters could help investigating and quantifying these dependencies. The time courses obtained in this study show the expected characteristics of VASO, ASL, and BOLD (see Figure 3) (9). The increased CBV-weighted signal changes occurring during functional activation can be explained by the increased SNR of SS-SI-VASO at ultrahigh field compared to classic VASO, as well as reduced partial volume effects as a result of the high spatial resolution used $(17,33)$. However, comparable changes in VASO signal have already been found in earlier studies, even at lower field strengths $(20,34)$. The VASO signal is acquired at blood nulling time and therefore reflects the amount of gray matter signal in each voxel. During functional activation of a specific brain area, the blood vessels dilate and therefore reduce the remaining gray matter signal in these voxels, leading to a negative VASO signal. BOLD and ASL signal changes are positive, as expected. The signal time courses of all three quantities are as expected for single subject and group analysis.

\section{CONCLUSION}

In this study, we have introduced a modified version of the MRI sequence introduced by Yang et al. (9) that enables simultaneous acquisition of CBV-, CBF-, and BOLDweighted MR signals at 7 Tesla. The MR pulse sequence used in this study was optimized for use at ultra-high field, but with appropriate adjustment of the timing parameters is equally applicable at clinical field strengths, albeit with lower SNR. Functional activation maps and time courses of CBV-, CBF-, and BOLD-weighted MRI signals obtained through this technique demonstrated the applicability of this novel approach. Simultaneous acquisition of VASO, ASL, and BOLD-based MRI signals can be a powerful tool for functional studies of brain function and a time efficient alternative for cognitive studies. Future studies could use our technique to investigate neurovascular coupling and its changes across diseases, age, and pharmacology. Furthermore, calibrated BOLD studies are expected to benefit from additional information about changes in CBV and overcome uncertainties related to the flow-volume coupling coefficient.

\section{REFERENCES}

1. Ogawa S, Lee TM, Kay AR, Tank DW. Brain magnetic resonance imaging with contrast dependent on blood oxygenation. Proc Natl Acad Sci U S A 1990;87:9868-9872.

2. Turner R. How much cortex can a vein drain? Downstream dilution of activation-related cerebral blood oxygenation changes. Neuroimage 2002;16:1062-1067.

3. Lu H, Zhao C, Ge Y, Lewis-Amezcua K. Baseline blood oxygenation modulates response amplitude: physiologic basis for intersubject variations in functional MRI signals. Magn Reson Med 2008;60:364-372.

4. Kim SG. Quantification of relative cerebral blood flow change by flow-sensitive alternating inversion recovery (FAIR) technique: application to functional mapping. Magn Reson Med 1995;34:293-301.

5. Wong EC, Buxton RB, Frank LR. Implementation of quantitative perfusion imaging techniques for functional brain mapping using pulsed arterial spin labeling. NMR Biomed 1997;10:237-249.

6. Dai W, Garcia D, de Bazelaire C, Alsop DC. Continuous flow-driven inversion for arterial spin labeling using pulsed radio frequency and gradient fields. Magn Reson Med 2008;60:1488-1497. 
7. Detre JA, Wang J. Technical aspects and utility of fMRI using BOLD and ASL. Clin Neurophysiol Off J Int Fed Clin Neurophysiol 2002; 113:621-634.

8. Lu H, Golay X, Pekar JJ, Van Zijl PCM. Functional magnetic resonance imaging based on changes in vascular space occupancy. Magn Reson Med 2003;50:263-274.

9. Yang Y, Gu H, Stein EA. Simultaneous MRI acquisition of blood volume, blood flow, and blood oxygenation information during brain activation. Magn Reson Med 2004;52:1407-1417.

10. Gu H, Stein EA, Yang Y. Nonlinear responses of cerebral blood volume, blood flow and blood oxygenation signals during visual stimulation. Magn Reson Imaging 2005;23:921-928.

11. Lin A-L, Fox PT, Yang Y, Lu H, Tan L-H, Gao J-H. Evaluation of MRI models in the measurement of CMRO2 and its relationship with CBF. Magn Reson Med 2008;60:380-389.

12. Lin A-L, Fox PT, Yang Y, Lu H, Tan L-H, Gao J-H. Time-dependent correlation of cerebral blood flow with oxygen metabolism in activated human visual cortex as measured by fMRI. Neuroimage 2009;44:16-22.

13. Gu H, Lu H, Ye FQ, Stein EA, Yang Y. Noninvasive quantification of cerebral blood volume in humans during functional activation. Neuroimage 2006;30:377-387.

14. Glielmi CB, Schuchard RA, Hu XP. Estimating cerebral blood volume with expanded vascular space occupancy slice coverage. Magn Reson Med 2009;61:1193-1200.

15. Ciris PA, Qiu M, Constable RT. Noninvasive MRI measurement of the absolute cerebral blood volume-cerebral blood flow relationship during visual stimulation in healthy humans. Magn Reson Med 2014;72: 864-875.

16. Ciris PA, Qiu M, Constable RT. Non-invasive quantification of absolute cerebral blood volume during functional activation applicable to the whole human brain. Magn Reson Med 2013.

17. Huber L, Ivanov D, Krieger SN, Streicher MN, Mildner T, Poser BA, Möller HE, Turner R. Slab-selective, BOLD-corrected VASO at 7 Tesla provides measures of cerebral blood volume reactivity with high signal-to-noise ratio. Magn Reson Med 2014;72:137-148.

18. Hurley AC, Al-Radaideh A, Bai L, Aickelin U, Coxon R, Glover P, Gowland PA. Tailored RF pulse for magnetization inversion at ultrahigh field. Magn Reson Med 2010;63:51-58.

19. Lu H, van Zijl PCM. Experimental measurement of extravascular parenchymal BOLD effects and tissue oxygen extraction fractions using multi-echo VASO fMRI at 1.5 and 3.0 T. Magn Reson Med 2005;53:808-816.

20. Hua J, Jones CK, Qin Q, van Zijl PCM. Implementation of vascularspace-occupancy MRI at 7T. Magn Reson Med 2013;69:1003-1013.
21. Huber L, Goense J, Kennerley AJ, Ivanov D, Krieger SN, Lepsien J, Trampel R, Turner R, Möller HE. Investigation of the neurovascular coupling in positive and negative BOLD responses in human brain at $7 \mathrm{~T}$. Neuroimage 2014;97:349-362.

22. Buxton RB. Interpreting oxygenation-based neuroimaging signals: the importance and the challenge of understanding brain oxygen metabolism. Front Neuroenergetics 2010;2:8.

23. Goense J, Merkle H, Logothetis NK. High-resolution fMRI reveals laminar differences in neurovascular coupling between positive and negative BOLD responses. Neuron 2012;76:629-639.

24. Jin T, Kim S-G. Improved cortical-layer specificity of vascular space occupancy fMRI with slab inversion relative to spin-echo BOLD at 9.4 T. Neuroimage 2008;40:59-67.

25. Wong EC, Buxton RB, Frank LR. Quantitative imaging of perfusion using a single subtraction (QUIPSS and QUIPSS II). Magn Reson Med 1998;39:702-708.

26. Garcia DM, Duhamel G, Alsop DC. Efficiency of inversion pulses for background suppressed arterial spin labeling. Magn Reson Med 2005; 54:366-372.

27. Ishimori Y, Monma M, Kawamura H, Miyata T. Time spatial labeling inversion pulse cerebral MR angiography without subtraction by use of dual inversion recovery background suppression. Radiol Phys Technol 2011;4:78-83.

28. Aguirre GK, Zarahn E, D'esposito M. The variability of human, BOLD hemodynamic responses. Neuroimage 1998;8:360-369.

29. Duff E, Xiong J, Wang B, Cunnington R, Fox P, Egan G. Complex spatio-temporal dynamics of fMRI BOLD: A study of motor learning. Neuroimage 2007;34:156-168.

30. Liu P, Hebrank AC, Rodrigue KM, Kennedy KM, Section J, Park DC, Lu H. Age-related differences in memory-encoding fMRI responses after accounting for decline in vascular reactivity. Neuroimage 2013; 78:415-425.

31. Poser BA, van Mierlo E, Norris DG. Exploring the post-stimulus undershoot with spin-echo fMRI: implications for models of neurovascular response. Hum Brain Mapp 2011;32:141-153.

32. Uludağ K. Transient and sustained BOLD responses to sustained visual stimulation. Magn Reson Imaging 2008;26:863-869.

33. Pfeuffer J, Adriany G, Shmuel A, Yacoub E, Van De Moortele P-F, $\mathrm{Hu}$ X, Ugurbil K. Perfusion-based high-resolution functional imaging in the human brain at 7 Tesla. Magn Reson Med 2002;47:903911

34. Hua J, Qin Q, Donahue MJ, Zhou J, Pekar JJ, van Zijl PCM. Inflowbased vascular-space-occupancy (iVASO) MRI. Magn Reson Med 2011;66:40-56. 